Original Article

Prabin Shrestha, MD, PhD

Department of neuroscience

B\&B Hospital, Gwarko

Lalitpur, Nepal

Anish M Singh, MS

Department of neuroscience

B\&B Hospital, Gwarko

Lalitpur, Nepal

Address for correspondence:

Prabin Shrestha, MD, PhD

Department of neuroscience

B\&B Hospital, Gwarko

Lalitpur, Nepal

Email: prabinshrestha@hotmail.com

Received, 15 October, 2016

Accepted, 9 November, 2016

$\mathrm{P}$ ituitary adenoma is one of the common brain tumors after glioma, meningioma and acoustic tumors. Pituitary tumor was first identified by Pierre Marie, a French neurologist, in 1886. He studied
Nepal Journal of Neuroscience 13:63-67, 2016

\section{Pituitary Adenoma Surgery: Retrospective Analysis of My Personal Experience}

Pituitary tumors are one of the common brain tumors after gliomas, meningieomas and acoustic schwaanomas. It is commonly encountered in Nepal also as in other countries.

Retrospective case series analytical study was done. The cases of pituitary adenoma from 2009 till 2015 were included. Types of pituitary adenoma, surgical technique, postoperative morbidities, mortalities and outcome were analyzed.

Total 21 cases of pituitary adenoma were operated. All the cases were diagnosed by MRI, plain and contrast. In addition, thorough hormonal evaluation was done. All the cases were operated via transnasal transsphenoidal (TSS) approach. Microscope was used in all the cases whereas endoscope was used in selected cases. Preoperative lumbar puncture and CSF drain was not done in any case.

Postoperative transient nasal CSF leak and diabetes insipidus were the most common surgery related morbidities in almost $50 \%$ cases which were totally cured with time. All the patients became asymptomatic and got better after surgery. Postoperative mortality and long term morbidity were nil. Recurrence was noted in one case after 3 years of surgery.

Pituitary tumor surgery is safe and rewarding provided it is done carefully with skilled hands. TSS is a good approach with minimal postoperative complications. Better to have both microscope and endoscope for TSS.

Key Words: pituitary adenoma, surgery, TSS

the case of acromegaly and postulated that it is related with pituitary gland. ${ }^{9}$

Pituitary tumors constitute about $10-15 \%$ of total intracranial tumors out of which about $90 \%$ are adenoma. 


\section{Shrestha et al}

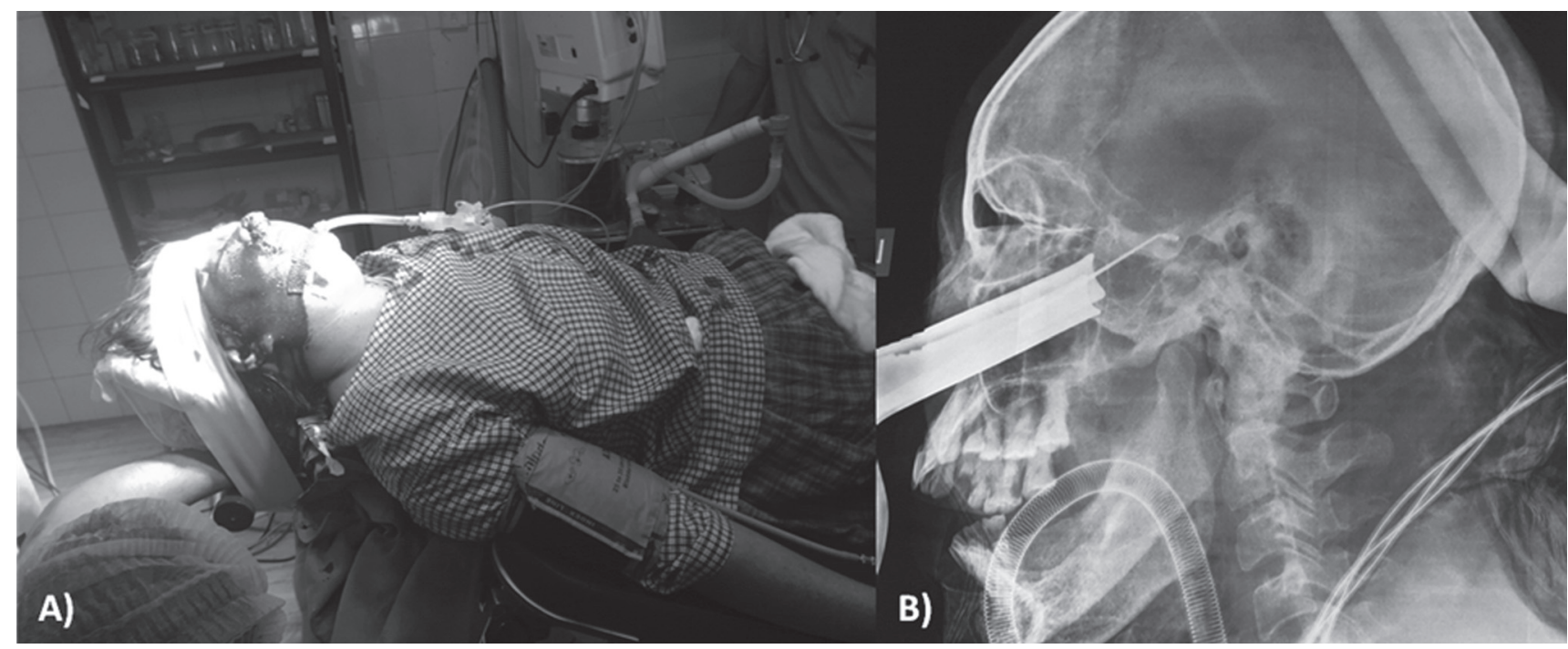

Figure 1: Intraoperative pictures, A) positioning of patient, supine with about 20 degree head up, B) intraoperative check $X$-ray to confirm the anatomical location

Dectection of putitary tumors has significantly increased in recent decades with the advent of CT scan and MRI.

Clinical manifestation of pituitary tumors are due to distant endocrinal effect of pituitary hormones and due to local mass effect of pituitary adenoma in supra and parasellar region.

On the basis of size of tumor, pituitary adenoma can be divided into mircoadenoma if it is $<10 \mathrm{~mm}$ in size and macroadenoma if $>10 \mathrm{~mm}$ in size. It is further called giant adenoma if $>20 \mathrm{~mm}$ in size. On the basis of hormonal secretion, pituitary tumors again can be divided into functioning and non-functioning. Moreover, non functioning pituitary tumors can affect hormone producing cells due to its local mass effect and can cause underproduction of certain hormones leading to hypopituitarism.

\section{Materials and Methods}

This is a retrospective case series analytical study of pituitary adenoma that underwent surgery from 2009 till 2015 in Norvic international hospital, Kathmandu and B\&B hospital, Lalitpur. Total 21 cases of pituitary adenoma cases were operated in above mentioned period. All the cases were diagnosed by plain and contrast MRI of brain and hormonal analysis including serum prolactin, growth hormone, thyroid hormones and cortisole. In addition, other hormones like follicular stimulating hormone, leutinizing hormone etc were also checked in some cases.

The main objective of this study is to assess the outcome and share the knowledge and experience of pituitary surgery in Nepalese context.

\section{Surgical Procedure}

All the cases were operated via transsphenoidal approach (TSS), ie non of the cases were operated by transcranial approach. Moreover, all the cases were operated by transnasal route using right nostril, not by sublabial route. All the cases were operated by using microscope. In some cases endoscope was also used and in some cases, due to various reasons, not.

Patient was placed in supine position with head elevated to about 20 degree and tilted to left. Slight neck extension was done so that right nostril was directed straight from surgeons vision (Figure 1 A).

After injecting local anesthetic agent in the nasal mucosa, mucosal incision was given at the junction of vomer bone and cartilaginous part of nasal septum. Vomer was taken out and sphenoid sinus opened sufficiently to exposed whole of the sella floor. Check x-ray was taken to confirm the approach (Figure 1 B). Sella was opened and check ex-ray was taken once again, if needed, to confirm the required anatomical location. Dura was opened and tumor resected with the help of suction cannula and ring curette of different size and angle. Diaphragm sella, arachnoid layer, was exposed most often before completion of surgery (Figure 2). Sella was packed with fatty tissue and nasal cavity was packed on both sides with ribbon gauze soaked with antibacterial ointment.

\section{Results}

Age of patients in our experience ranged from 24 to 65 years. Female patients comprised $75 \%$ (15/21) and male patients comprised $25 \%(6 / 21)$.

Of 21 cases, 5 cases were nonfunctioning pituitary 


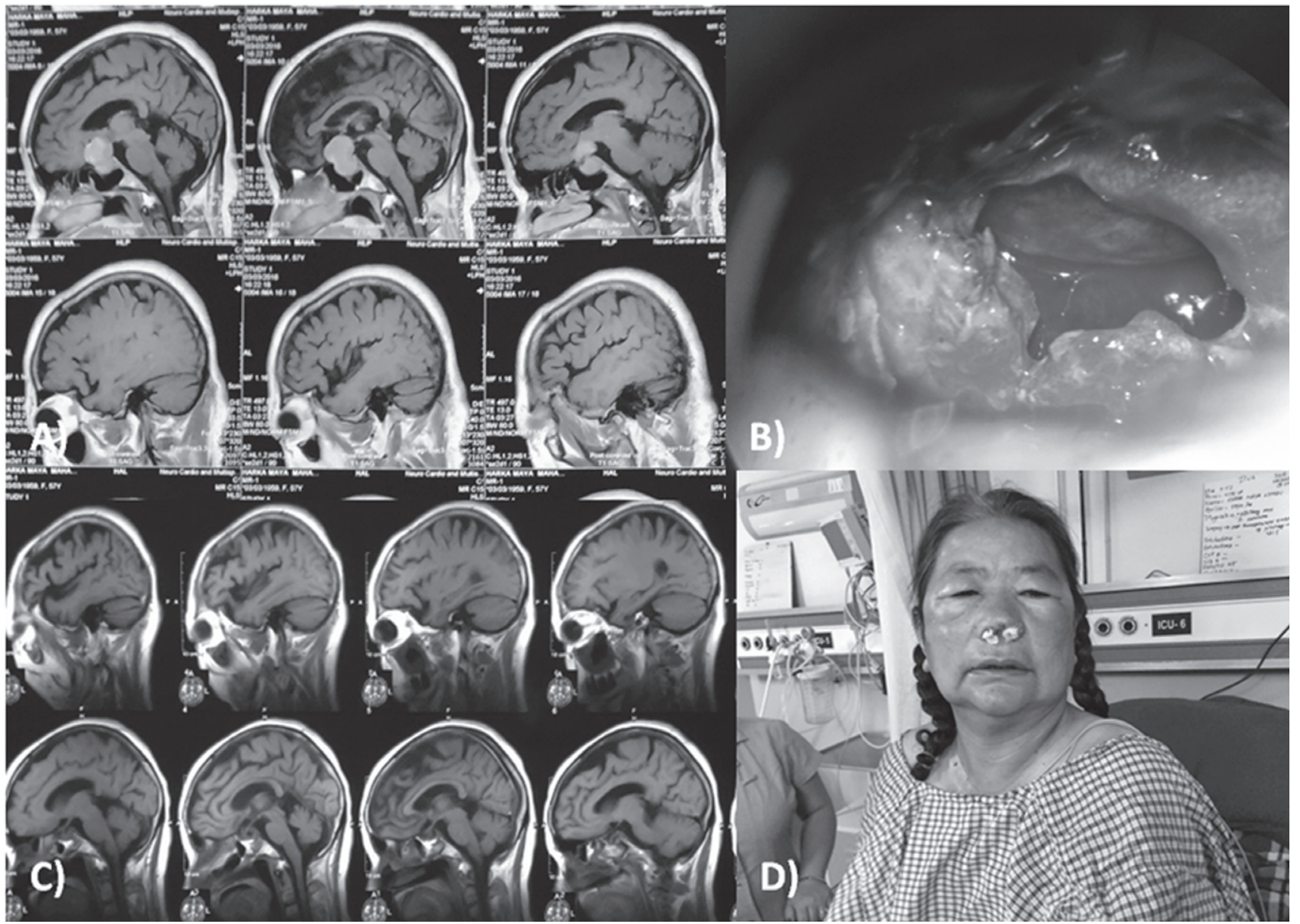

Figure 2) Illustration 1, A) pre-op MRI brain T1 contrast sagittal image showing huge pituitary adenoma, B) intra operative picture showing exposure of diaphragm sella and arachnoid layer after complete resection of pituitary tumor, C) Post operative MRI brain T1 plain sagittal image showing complete resection of tumor, D) patient on $3^{\text {rd }}$ post operative day

adenomas and remaining 16 cases were functioning. Of 16 nonfunctioning adenomas, 11 cases were prolactinomas and remaining 5 cases were acromegaly. Not a single case in our experience was a malignant pituitary adenoma.

Out of total cases, 12 cases were macroadenoma and 9 cases were microadenoma.

Post operatively, CSF rhinorrhoea was noticed in $8 / 21$ cases $(38 \%)$ which was more frequent in the initial days of surgical experience and became less frequent afterwards. Similarly diabetes insipidus (DI) developed in 9/21 cases $(42 \%)$. DI was also more frequent in initial days and became less frequent afterwards like CSF rhinorrhoea. There was recurrence of one case after 3 years of first surgery which was reoperated via same route. She had subtotal resection of tumor in the first surgery. Hormonal correction was achieved in most of the cases. There was not a single case of surgery related chronic morbidity or mortality. Similarly, there was not a single case of pituitary apoplexy either.
Total hospital stay was 3 to 10 days, the average being 6 days.

As shown by post operative CT and MRI, total or near total resection was achieved in more than $60 \%$ of cases and subtotal resection was achieved in less than $40 \%$ of cases.

\section{Discussion}

Pituitary adenoma is a common neoplastic lesion of brain. ${ }^{9}$ Transsphenoidal surgery is the main line of treatment for many kinds of pituitary adenoma. ${ }^{1}$

Medical management like Dopamine agonist and radiotherapy are other modalities of its treatment. Dopamin agonist is a good drug for the conservative management of prolactinomas. It can be tried in cases which are not indicated for surgery. Studies have shown that it helps significantly in lowering the level of prolactin irrespective of size of tumor. ${ }^{12}$ In our practice we also treat 


\section{Shrestha et al}

the cases of hyperprolactinemia with or without adenoma with Dopamin agonist such as Bromocryptin and have found the same result. However, those with bigger size are treated surgically to prevent and cure the mass effect. Though no correct data is available in our study, dopamine agonist like Bromocryptine has been used in several cases before and after surgery. We haven't used radiotherapy for any of our patients with pituitary adenoma.

Most of our cases presented with hormonal disturbance and mass effect leading to visual impairment. However, there was not a single case presenting with apoplexy. ${ }^{13}$

Even though it is found in any age and sex, it has been more commonly seen in females than in males. ${ }^{10}$ Our study also showed the same. Three fourth of our cases were female. It is a slow growing tumor and becomes big enough when first becomes symptomatic. Therefore, macroadenoma is more commonly found as shown by Wang JN et al and same did our study. ${ }^{10}$ Most of the pituitary adenomas can be and are operated by TSS approach as shown by other studies and our study. ${ }^{10}$ Recurrence of tumor is uncommon as seen in our case.

Cystic prolactinoma was relatively uncommon as shown by Ogiwara T et al. ${ }^{4}$ They had 5/28 cases of cystic prolactinoma where as in our case we had only one out of 21 cases. Surgical resection gives the complete cure as in our case.

Regarding surgical technique, there are mainly endoscopic and microscopic tenchniques and both of them are used via endonasal TSS approach. Recent studies have shown that endoscopic technique is better in terms of extent of tumor resection. ${ }^{5,7,8}$ For smaller intrasellar tumors both techniques have been found equally good without much difference. However, for larger and parasellar tumors, endoscopic techniques have been found to be much helpful. Endoscopic technique especially has been found to reduce the total surgical time, incidence of CSF leak and increase the extent of tumor resection. Endoscopic technique is also helpful in recurrent and residual cases. ${ }^{3}$ However, there seems pros and cons of both the techniques. We used microsurgical technique in all the cases, except in few where we used both, due to lack of appropriate endoscopes.

Visual field defect is a major indication for surgical removal. Studies have shown significant visual improvement after surgical removal of pituitary tumors. ${ }^{2,11}$ Though we haven't done thorough investigation of vision before and after surgery, our experience also showed the same thing. In addition, studies have shown that their overall health and quality of life improved significantly after surgical removal of the tumor.

Regarding post operative complications, CSF leak is the most commonly encountered complications. One study has shown that CSF leak was found in about one third of the cases. ${ }^{6}$ We also experienced the same thing. However, in our case incidence of CSF leak was reduced significantly as we gained more and more experience. Besides, subdural hematoma has also been found to occur on and off which we haven't experienced so far. It has been found that post operative subdural hematoma was associated with intraoperative CSF leak. ${ }^{6}$

\section{Conclusion}

Even though our experience is small, it looks satisfactory as far as surgical technique, its complications and ultimate outcome is concerned. Lack of endoscope for pituitary surgery and hormonal assessment before and after surgery in detail are few limitations in our part.

\section{Reference}

1. Buchfelder M, Schlaffer SM. The surgical treatment of acromegaly. Br J Neurosurg 30:637-642, 2016

2. Lee J, Kim SW, Kim DW, et al. Predictive model for recovery of visual field after surgery of pituitary adenoma. J Neurooncol 130:155-164, 2016

3. Negm HM, Al-Mahfoudh R, Pai M, et al. Reoperative endoscopic endonasal surgery for residual or recurrent pituitary adenomas. J Neurosurg, 2016 [Epub ahead of print]

4. Ogiwara T, Horiuchi T, Nagm A, Goto T, Hongo K. Significance of surgical management for cystic prolactinoma. Pituitary, 2016 [Epub ahead of print]

5. Phan K, Xu J, Reddy R, Kalakoti P, Nanda A, Fairhall J. Endoscopic endonasal versus microsurgical transphenoidal approach for growth hormonesecreting pituitary adenomas - systematic review and meta-analysis. World Neurosurg, 2016 [Epub ahead of print]

6. Takeuchi K, Watanabe T, Nagatani T, Nagata Y, Chu J, Wakabayashi T. Incidence and risk factors of subdural hematoma after intraoperative cerebrospinal fluid leakage during the transsphenoidal approach. Pituitary 9:565-572, 2016

7. Qureshi T, Chaus F, Fogg L, Dasgupta M, Straus D, Byrne RW. Learning curve for the transsphenoidal endoscopic endonasal approach to pituitary tumors. Br J Neurosurg 30:637-642, 2016

8. Singh H, Essayed WI, Cohen-Gadol A, Zada G, Schwartz TH. Resection of pituitary tumors: endoscopic versus microscopic. J Neurooncol 130:309-317, 2016 
Pituitary Adenoma

9. Villwock JA, Villwock M, Deshaies E, Goyal P. Significant increases of pituitary tumors and resections from 1993 to 2011. Int Forum Allergy Rhinol 4:767-770, 2014

10. Wang JN, Wang LX, Lian W, et al. Surgical treatment of pituitary adenomas in childhood and adolescence. Zhonghua Yi Xue Za Zhi 96:2998-3002, 2016

11. WolfA, Coros A, Bierer J, et al. Quantitative evaluation of vision-related and health-related quality of life after endoscopic transsphenoidal surgery for pituitary adenoma. J Neurosurg, 2016 [Epub ahead of print]

12. Yedinak CG, Cetas I, Ozpinar A, McCartney S, Dogan A, Fleseriu M. Dopamine agonist therapy induces significant recovery of HPA axis function in prolactinomas independent of tumor size: a large single center experience. Endocrine 54:191-197, 2016

13. Zhang RC, Mu YF, Dong J, Lin XQ, Geng DQ. Complex effects of apoplexy secondary to pituitary adenoma. Rev Neurosci 2016 [Epub ahead of print] 